\title{
Differential Regularization of Chern-Simons-Maxwell Spinor and Scalar Electrodynamics
}

\author{
M. Chaichian ${ }^{a, b}$, W. F. Chen ${ }^{b \dagger}$ and H.C. Lee ${ }^{c}$ \\ ${ }^{a}$ High Energy Physics Division, Department of Physics, Univeristy of Helsinki \\ ${ }^{b}$ Helsinki Institute of Physics, P.O. Box 9 (Siltavuorenpenger 20 C) \\ FIN-00014 University of Helsinki, Finland \\ ${ }^{c}$ Department of Physics, National Central University, Chungli, Taiwan 320, ROC
}

\begin{abstract}
Differential regularization is used to investigate the one-loop quantum corrections to Chern-Simons-Maxwell spinor and scalar electrodynamics. We illustrate the techniques to write the loop amplitudes in coordinate space. The short-distance expansion method is developed to perform the Fourier transformation of the amplitudes into momentum space and the possible renormalization ambiguity in Chern-Simons type gauge theories in terms of differential regularization is discussed. We also stress that the surface terms appearing in the differential regularization should be kept along for finite theories and they will result in the finite renormalization ambiguity.
\end{abstract}

Differential regularization is a relatively new regularization scheme [1]. The basic idea of this regularization is quite simple. It works in coordinate space and is based on the observation that the UV divergence reflects in the fact that the higher order amplitude can not have a Fourier transform into momentum space due to the short-distance singularity. Thus one can regulate such an amplitude by first writing its singular parts as the derivatives of the

\footnotetext{
†ICSC-World Laboratory, Switzerland
} 
normal functions, which have well defined Fourier transformation, and then by performing the Fourier transformation in partial integration and discarding the surface term, in this way one can directly get the renormalized result. This regularization scheme successfully avoids the ambiguities of the dimensional regularization in defining the dimensional continuation of $\gamma_{5}$-like objects since it does not need to continue the dimension of space-time. Up to now this method has almost been verified in almost every field theory including the supersymmetric one [1 5]. Its relation with the conventional dimensional regularization and the compatibility with unitary at two-loop level have also been investigated [6 8]. In some cases it indeed has advantages over all the conventional regularization schemes. Especially, it is very convenient to use this regularization to discuss the conformal properties of quantum field theory 91 .

In this letter we use this regularization to investigate the one-loop quantum correction to Chern-Simons-Maxwell scalar and spinor electrodynamics [10]. One straightforward reason, as mentioned above, is that it avoids the ambiguity of dimensional continuation in defining the three-dimensional completely antisymmetric tensor $\epsilon_{\mu \nu \rho}$. As we know, the dimensionality in Chern-Simons-type theory plays an important role since Chern-Simons term is a topological one and the topological properties of theory depend heavily on the three-dimensional antisymmetric tensor, thus a calculation without using dimensional continuation is called for. The main motivation is that we want to explore the possible origin of renormalization ambiguity of perturbative Chern-Simons theory in the framework of dimensional regularization. This ambiguity depends on the concrete regularization schemes [11-13] and is the most puzzled feature of Chern-Simons type theories, up to now it has not been well understood. Therefore, it is desirable to work in a regularization scheme which does not greatly change the original theory. Indeed, it has been found that higher covariant derivatived Pauli-Villars regularization can bring non-physical quantum corrections [14], or at least this regularization does not return back to the original theory when the regulator is removed [15]. We believe that up to now differential regularization is the most appropriate method in this aspect since it does not change the Lagrangian of the theory explicitly. Furthermore, from 
the view point of practical calculations, this regularization is very suitable for the threedimensional quantum field theory since the propagators in three-dimensional space-time takes a very simple form. In particular, the short-distance expansion technique is developed in Ref. [16], which can be used to calculate the one-loop quantum corrections exactly.

The Lagrangian in Euclidean space is as following

$$
\mathcal{L}=-\frac{1}{4 \lambda} F_{\mu \nu} F_{\mu \nu}-\frac{i k}{8 \pi} \epsilon_{\mu \nu \rho} A_{\mu} \partial_{\nu} A_{\rho}-\frac{1}{2 \alpha}\left(\partial_{\mu} A_{\mu}\right)^{2}-\mathcal{L}_{\text {matter }}
$$

where

$$
\mathcal{L}_{\text {matter }}=D_{\mu} \phi^{+} D_{\mu} \phi+m^{2} \phi^{+} \phi
$$

for scalar field and

$$
\mathcal{L}_{\text {matter }}=\bar{\psi}\left(\gamma_{\mu} D_{\mu}+i m\right) \psi
$$

for the spinor case. The $\gamma$ matrices are defined as

$$
\gamma_{\mu}=i \sigma_{\mu}, \quad \gamma_{\mu} \gamma_{\nu}=-\delta_{\mu \nu}-\epsilon_{\mu \nu \rho} \gamma_{\rho}, \quad \operatorname{Tr}\left(\gamma_{\mu} \gamma_{\nu}\right)=-2 \delta_{\mu \nu}
$$

The propagators for electron, scalar field and gauge field take very simple forms in coordinate space:

$$
\begin{gathered}
S(x)=\left(\gamma_{\mu} \partial_{\mu}-i m\right) \frac{1}{4 \pi} \frac{1}{x} e^{-m x}, \\
D(x)=\frac{1}{4 \pi} \frac{1}{x} e^{-m x} \\
G_{\mu \nu}(x)=\left[\frac{i}{k /(4 \pi)} \epsilon_{\mu \nu \rho} \partial_{\rho}-\frac{\lambda}{n^{2}}\left(\partial^{2} \delta_{\mu \nu}-\partial_{\mu} \partial_{\nu}\right)\right] \frac{1}{4 \pi} \frac{1}{x}\left(1-e^{-n x}\right),
\end{gathered}
$$

where (and in what follows) $x \equiv|x|, n \equiv \frac{\lambda k}{4 \pi}$ and we work in Lauge gauge $(\alpha=0)$.

Now we calculate the vacuum polarization tensor. Let us first see the contribution from electron loop:

$$
\begin{aligned}
\Pi_{\mu \nu}^{(\text {spinor })}(x) & =-\operatorname{Tr}\left[\Gamma_{\mu} S(x) \Gamma_{\nu} S(-x)\right] \\
& =-\frac{e^{2}}{8 \pi^{2}}\left[2 x_{\mu} x_{\nu}\left(\frac{1}{x^{6}}+\frac{2 m}{x^{5}}+\frac{m^{2}}{x^{4}}\right) e^{-2 m x}\right.
\end{aligned}
$$




$$
\begin{aligned}
& -\delta_{\mu \nu}\left(\frac{1}{x^{4}}+\frac{2 m}{x^{3}}+\frac{2 m^{2}}{x^{2}}\right) e^{-2 m x} \\
& \left.-2 i m \epsilon_{\mu \nu \rho} \partial_{\rho}\left(\frac{1}{x} e^{-m x}\right) \frac{1}{x} e^{-m x}\right] .
\end{aligned}
$$

Obviously, the terms $1 / x^{n}$ with $n \geq 3$ can not perform their Fourier transformation into momentum space. According to the idea of differential regularization, the vacuum polarization tensor can be written as the differential regulated version

$$
\begin{aligned}
\Pi_{\mu \nu}^{(\text {spinor })}(x) & =-\frac{e^{2}}{8 \pi^{2}}\left(\partial_{\mu} \partial_{\nu}-\delta_{\mu \nu} \partial^{2}\right)\left[\left(\frac{1}{4} \frac{1}{x^{2}}+\frac{1}{2} \frac{m}{x}\right) e^{-2 m x}+m^{2} \operatorname{Ei}(-2 m x)\right] \\
& +\frac{i e^{2}}{8 \pi^{2}} m \epsilon_{\mu \nu \rho} \partial_{\rho}\left(\frac{1}{x^{2}} e^{-2 m x}\right)
\end{aligned}
$$

with $\operatorname{Ei}(y)=\int_{y}^{\infty} e^{-t} t^{-1} d t$ being the exponential integral function. One can see that there is no new dimensional parameter appearing in Eq.(7), which means the finiteness of the vacuum polarization tensor. As suggested in Ref. [1] and developed in Ref. [16], we use the short-distance technique to perform Fourier transformation into momentum space so that we can preserve the possible nonvanishing surface term. With aid of the formulas

$$
\begin{aligned}
\int_{R_{\epsilon}^{3}} d^{3} x \partial_{\mu} f(x) e^{i p \cdot x} & =4 \pi i \frac{\partial}{\partial p_{\mu}}\left[\frac{\sin (p \epsilon)}{p}\right] f(\epsilon)-i p_{\mu} \int_{R_{\epsilon}^{3}} d^{3} x e^{i p \cdot x} f(x) \\
\int_{R_{\epsilon}^{3}} e^{i p \cdot x} \partial_{\mu} \partial_{\nu} f(x) & =\left.4 \pi \frac{\partial^{2}}{\partial p_{\mu} \partial p_{\nu}}\left[\frac{\sin (p \epsilon)}{p \epsilon}\right] \frac{d}{d x} f(x)\right|_{x=\epsilon}-4 \pi p_{\mu} \frac{\partial}{\partial p_{\nu}}\left[\frac{\sin (p \epsilon)}{p}\right] f(\epsilon) \\
& -p_{\mu} p_{\nu} \int_{R_{\epsilon}^{3}} d^{3} x f(x) e^{i p \cdot x}
\end{aligned}
$$

where $p \equiv|p|, R_{\epsilon}^{3}$ denotes the integration region $R^{3}-B_{\epsilon}$ and $B_{\epsilon}$ is a small sphere of radius $\epsilon$ around the origin, we obtain

$$
\begin{aligned}
\Pi_{\mu \nu}^{(\text {spinor })}(p) & =\frac{e^{2}}{2 \pi}\left\{\epsilon_{\mu \nu \rho} p_{\rho} \frac{m}{p} \arctan \frac{p}{2 m}-\left(p^{2} \delta_{\mu \nu}-p_{\mu} p_{\nu}\right)\left[\frac{m}{2 p^{2}}\right.\right. \\
& \left.\left.+\frac{1}{4}\left(\frac{m}{p}-\frac{4 m^{2}}{p^{3}}\right) \arctan \frac{p}{2 m}\right]\right\} .
\end{aligned}
$$

In deriving (9) we have taken the limit $\epsilon \rightarrow 0$ after performing the integration. The necessity of preserving the surface term should be stressed for a finite theory. Otherwise, if one 
throws away the surface term (as that suggested in the original paper [1] on differential regularization), some finite terms as $\epsilon \rightarrow 0$ will lose, this will certainly lead to the ambiguity of finite renormalization.

The vacuum polarization tensor in scalar case is a little complicated since there is derivative on the vertex, but the calculation is straightforward. It reads as

$$
\begin{aligned}
\Pi_{\mu \nu}^{(\text {scalar })}(x) & =e^{2}\left[\partial_{\mu} D(-x) \partial_{\nu} D(x)-D(x) \partial_{\mu} \partial_{\nu} D(-x)-D(-x) \partial_{\mu} \partial_{\nu} D(x)+\partial_{\mu} D(x) \partial_{\nu} D(-x)\right] \\
& =\frac{e^{2}}{8 \pi^{2}}\left\{\frac{x_{\mu} x_{\nu}}{x^{2}}\left[\frac{d}{d x} D(x)\right]^{2}-\frac{x_{\mu} x_{\nu}}{x} D(x) \frac{d}{d x}\left[\frac{1}{x} \frac{d}{d x} D(x)\right]-D(x) \frac{\delta_{\mu \nu}}{x} \frac{d}{d x} D(x)\right\} \\
& =\frac{e^{2}}{8 \pi^{2}}\left[\delta_{\mu \nu}\left(\frac{1}{x^{4}}+\frac{m}{x^{3}}\right) e^{-2 m x}-x_{\mu} x_{\nu}\left(\frac{2}{x^{6}}+\frac{m}{x^{5}}\right) e^{-2 m x}\right] \\
& =\frac{e^{2}}{16 \pi^{2}}\left(\partial_{\mu} \partial_{\nu}-\delta_{\mu \nu} \partial^{2}\right)\left[-\frac{1}{2 x^{2}} e^{-2 m x}+\frac{m}{x} e^{-2 m x}+2 m^{2} \operatorname{Ei}(-2 m x)\right] .
\end{aligned}
$$

Its Fourier transformation is read as

$$
\Pi_{\mu \nu}^{(\text {scalar })}(p)=\frac{e^{2}}{4 \pi}\left(p^{2} g_{\mu \nu}-p_{\mu} p_{\nu}\right)\left[\frac{m}{p^{2}}-\frac{1}{2 p} \arctan \frac{p}{m}-\frac{2 m^{2}}{p^{3}} \arctan \frac{p}{2 m}\right] .
$$

The other one-loop two-point functions are self-energy of matter fields. The amplitude for the self-energy of electron is

$$
\begin{aligned}
\Sigma(x) & =\Gamma_{\nu} S(-x) \Gamma_{\mu}(x) G_{\mu \nu}(x) \\
& =\frac{e^{2}}{16 \pi^{2}}\left\{\frac{2 i}{k /(4 \pi)}\left[\left(\frac{1}{x^{4}}+\frac{m}{x^{3}}\right) e^{-m x}-\left(\frac{1}{x^{4}}+\frac{m+n}{x^{3}}+\frac{m n}{x^{2}}\right) e^{-(m+n) x}\right]\right. \\
& -\frac{2 m}{k /(4 \pi)} \gamma_{\rho} x_{\rho}\left[-\frac{1}{x^{4}} e^{-m x}+\left(\frac{1}{x^{4}}+\frac{n}{x^{3}}\right) e^{-(m+n) x}\right]+\frac{\lambda}{n^{2}} \gamma_{\mu} x_{\mu}\left[\left(\frac{4}{x^{6}}+\frac{4 m}{x^{5}}\right) e^{-m x}\right. \\
& +\left(-\frac{4}{x^{6}}-\frac{4(m+n)}{x^{5}}+\frac{-4 m n-2 n^{2}}{x^{4}}-\frac{2 m n^{2}}{x^{3}}\right) e^{-(m+n) x} \\
& \left.\left.-\frac{2 i m \lambda}{x^{2}} e^{-(m+n) x}\right]\right\}
\end{aligned}
$$

With similar operations as above, we write (12) as the differential regulated form

$$
\Sigma(x)=\frac{e^{2}}{16 \pi^{2}}\left\{\gamma _ { \mu } \partial _ { \mu } \left[-\frac{2 m}{k /(4 \pi)}\left(\left(\frac{1}{2 x^{2}}-\frac{m}{2 x}\right) e^{-m x}+\left(\frac{1}{2 x^{2}}+\frac{m-n}{2 x}\right) e^{-(m+n) x}\right.\right.\right.
$$




$$
\begin{aligned}
& \left.-\frac{m^{2}}{2} \operatorname{Ei}(-m x)+\frac{m^{2}-n^{2}}{2} \operatorname{Ei}[-(m+n) x]\right)-\lambda\left(\left(-\frac{1}{2 x^{2}}-\frac{m-n}{2 x}\right) e^{-(m+n) x}\right. \\
& \left.\left.+\frac{n^{2}-m^{2}}{2} \operatorname{Ei}[-(m+n) x]\right)\right]+\frac{\lambda}{n^{2}}\left[-\partial^{2}\left[\frac{1}{2} \frac{1}{x^{2}} e^{-m x}\left(1-e^{-n x}\right)\right]+\left(\frac{m^{2}}{x^{2}}-\frac{m^{3}}{2 x}\right) e^{-m x}\right. \\
& +\left(\frac{2 m^{2}-n^{2}}{2 x^{2}}-\frac{m^{2}(m+n)}{2 x}\right) e^{-(m+n) x}-m^{4} \operatorname{Ei}(-m x) \\
& \left.+\left(\frac{m^{4}}{2}-\frac{3 m^{2} n^{2}}{8}-\frac{n^{4}}{4}\right) \operatorname{Ei}[-(m+n) x]\right]+\frac{2 i}{k /(4 \pi)}\left[\partial^{2}\left[\frac{1}{2 x^{2}} e^{-m x}\left(1-e^{-n x}\right)\right]\right. \\
& \left.\left.-\frac{m^{2}}{2 x^{2}} e^{-m x}+\frac{m^{2}+n^{2}}{2 x^{2}} e^{-(m+n) x}\right]-\frac{2 i m \lambda}{x^{2}} e^{-(m+n) x}\right\}
\end{aligned}
$$

Using the short-distance expansion (8), we obtain the electron self-energy in momentum space as

$$
\begin{aligned}
\Sigma(p) & =\frac{e^{2}}{4 \pi} i \gamma_{\mu} p_{\mu}\left\{\frac { 2 m } { k / ( 4 \pi ) } \left[\frac{n}{2 p}+\left(\frac{1}{2}+\frac{m^{2}}{2 p^{2}}\right) \arctan \frac{p}{m}-\left(\frac{1}{2}+\frac{m^{2}-n^{2}}{2 p^{2}}\right) \arctan \frac{p}{m+n}\right.\right. \\
& -\lambda\left[\frac{m-n}{2 p}+\left(\frac{1}{2}-\frac{m^{2}-n^{2}}{2 p^{2}}\right) \arctan \frac{p}{m+n}\right]+\frac{\lambda}{n^{2}}\left[\frac{n}{2}+\frac{m^{3}}{2 p^{2}}\right. \\
& -\left(\frac{p}{2}+\frac{m^{2}}{p^{2}}-\frac{m^{4}}{2 p^{3}}\right) \arctan \frac{p}{m}+\left(\frac{p}{2}+\frac{m^{2}}{p}+\frac{n^{2}}{2 p}-\frac{m^{4}}{2 p^{3}}+\frac{3}{8} \frac{m^{2} n^{2}}{p^{3}}+\frac{n^{4}}{4 p^{3}}\right) \arctan \frac{p}{m+n} \\
& \left.\left.+\left(\frac{m^{4}}{2}-\frac{3 m^{2} n^{2}}{8}-\frac{n^{4}}{4}\right) \frac{m+n}{p^{2}\left[p^{2}+(m+n)^{2}\right]}-\frac{m^{2}(m-n)}{2\left[p^{2}+(m+n)^{2}\right]}\right]\right\} \\
& +\frac{e^{2}}{4 \pi}\left\{\frac{2 i}{k /(4 \pi)}\left[\frac{n}{2}-\left(\frac{p}{2}+\frac{m^{2}}{2 p}\right) \arctan \frac{p}{m}-\frac{m^{2}-n^{2}}{2 p} \arctan \frac{p}{m+n}\right]\right. \\
& \left.-\frac{2 i m \lambda}{p} \arctan \frac{p}{m+n}\right\} .
\end{aligned}
$$

As for the self-energy of the scalar field, since there are derivatives in the interaction vertex, usually it is very confusing to decide the derivative acting on external or internal lines when writing the amplitude in the coordinate space. The key technique is directly writing the amplitude in the Fourier transformed form and this can clearly show the action of the derivative in the vertex on external legs or internal lines,

$$
\begin{aligned}
\Omega(p) & =e^{2} \int d^{3} x e^{i p \cdot x}\left[i p_{\mu} \partial_{\nu} D(x) G_{\mu \nu}(x)+p_{\mu} p_{\nu} D(x) G_{\mu \nu}(x)\right. \\
& \left.-\partial_{\mu} \partial_{\nu} D(x) G_{\mu \nu}(x)+i p_{\nu} \partial_{\mu} D(x) G_{\mu \nu}(x)\right]
\end{aligned}
$$


Expanding each term in Eq.(15) and writing them in the derivative form, we obtain the scalar self-energy

$$
\begin{aligned}
& \Omega(p)=\frac{e^{2}}{16 \pi^{2}} \frac{\lambda}{n^{2}} \int d^{3} x e^{i p \cdot x} i p_{\mu} \partial_{\mu}\left\{\partial^{2}\left[\frac{1}{2 x^{2}}\left(e^{-m x}-e^{-(m+n) x}\right)\right]\right. \\
& +\left[\frac{m^{2}+n^{2}}{x^{2}}-\frac{(m-n)^{2}(m+n)}{2 x}\right] e^{-(m+n) x} \\
& \left.+\frac{m^{3}}{2 x} e^{-m x}+\frac{m^{4}}{2} \operatorname{Ei}(-m x)-\frac{\left(m^{2}-n^{2}\right)^{2}}{2} \operatorname{Ei}[-(m+n) x]\right\} \\
& +\frac{e^{2}}{16 \pi^{2}} \frac{\lambda}{n^{2}} \int d^{3} x e^{i p \cdot x} p_{\mu} p_{\nu}\left\{\partial _ { \mu } \partial _ { \nu } \left[\left(-\frac{m^{2}}{16}+\frac{3}{8 x^{2}}-\frac{5 m}{8 x}+\frac{m^{3} x}{16}\right) e^{-m x}\right.\right. \\
& +\left(\frac{(m-n)^{2}}{16}-\frac{3}{8 x^{2}}+\frac{5 m-3 n}{8 x}-\frac{x(m+n)(m-n)^{2}}{16}\right) e^{-(m+n) x} \\
& \left.+\left(-\frac{3 m^{2}}{4}+\frac{m^{4} x^{2}}{16}\right) \operatorname{Ei}(-m x)+\left(\frac{3 m^{2}-n^{2}}{4}-\frac{\left(m^{2}-n^{2}\right)^{2} x^{2}}{16}\right) \operatorname{Ei}[-(m+n) x]\right] \\
& +\delta_{\mu \nu}\left[\partial^{2}\left(-\frac{1}{8 x^{2}} e^{-m x}+\frac{1}{8 x^{2}} e^{-(m+n) x}\right)+\left(\frac{m^{2}}{4 x^{2}}-\frac{m^{3}}{8 x}\right) e^{-m x}\right. \\
& +\left(\frac{3 n^{2}-m^{2}}{4 x^{2}}+\frac{(m-n)^{2}(m+n)}{8 x}\right) e^{-(m+n) x} \\
& \left.\left.-\frac{m^{4}}{8} \operatorname{Ei}(-m x)+\frac{\left(m^{2}-n^{2}\right)^{2}}{8} \operatorname{Ei}[-(m+n) x]\right]\right\} \\
& +\frac{e^{2}}{16 \pi^{2}} \frac{\lambda}{n^{2}} \int d^{3} x e^{i p \cdot x}\left\{\partial ^ { 2 } \left[\partial^{2}\left(-\frac{1}{4 x^{2}} e^{-m x}+\frac{1}{4 x^{2}} e^{-(m+n) x}\right)+\frac{m^{2}}{2 x^{2}} e^{-m x}\right.\right. \\
& \left.\left.-\frac{m^{2}+n^{2}}{2 x^{2}} e^{-(m+n) x}\right]-\frac{m^{2} n^{2}}{2 x^{2}} e^{-(m+n) x}\right\} \text {. }
\end{aligned}
$$

After performing Fourier transformation through the short distance expansion, we have

$$
\begin{aligned}
\Omega(p) & =\frac{e^{2}}{4 \pi} \frac{\lambda}{n^{2}}\left\{\frac{m n(m+n)}{2}-\frac{7 n^{3}}{6}+n p^{2}-\frac{9 m^{5}}{8\left(p^{2}+m^{2}\right)}+\frac{5 m^{7}}{4\left(p^{2}+m^{2}\right)^{2}}-\frac{m^{9}}{2\left(p^{2}+m^{2}\right)^{3}}\right. \\
& +\frac{9(m-n)^{2}(m+n)^{3}}{8\left[p^{2}+(m+n)^{2}\right]}-\frac{5(m-n)^{2}(m+n)^{5}}{4\left[p^{2}+(m+n)^{2}\right]^{2}}+\frac{(m-n)^{2}(m+n)^{7}}{2\left[p^{2}+(m+n)^{2}\right]^{3}} \\
& +\left[-\frac{3 m^{4}}{8 p}-\frac{p^{3}}{2}-\frac{3 m^{4} p}{p^{2}+m^{2}}+\frac{6 m^{6} p}{\left(p^{2}+m^{2}\right)^{2}}-\frac{4 m^{8} p}{\left(p^{2}+m^{2}\right)^{3}}\right] \arctan \frac{p}{m}+\left[\frac{p^{3}}{2}\right. \\
& +\frac{\left[(m-n)^{2}+5\left(m^{2}+n^{2}\right)\right] p}{8}+\frac{\left(m^{2}-n^{2}\right)^{2}+2\left(m^{4}+n^{4}\right)}{8 p}+\frac{3\left(m^{2}-n^{2}\right)^{2} p}{8\left[p^{2}+(m+n)^{2}\right]}
\end{aligned}
$$




$$
\left.\left.-\frac{3(m-n)^{2}(m+n)^{4} p}{4\left[p^{2}+(m+n)^{2}\right]^{2}}+\frac{(m-n)^{2}(m+n)^{6} p}{2\left[p^{2}+(m+n)^{2}\right]^{3}}\right] \arctan \frac{p}{m+n}\right\} .
$$

Since the complete one-loop amplitude is already given, the finite renormalization can be easily performed by choosing a renormalization point (for example, one typical choice is $p=0$ ), as usual, we can define various renormalization constants and the radiative corrections.

In summary, we have shown how differential regularization can be used to investigate the one-loop two-point functions of three-dimensional Chern-Simons-Maxwell spinor and scalar electrodynamics. For the scalar case, where there is derivative on the vertex, we develop the technique to distinguish how the derivatives act on an external or an internal line properly when writing the amplitude in coordinate space. In particular, using the short-distance expansion technique, we show how a renormalization ambiguity can be generated for a finite theory. For example, in the Fourier transform of the term $\partial^{2}\left[\left(1-e^{-m x}\right) / x^{2}\right]$, if we consider the surface term through the short-distance expansion

$$
\int_{R_{\epsilon}^{3}} d^{3} x \partial^{2}\left(\frac{1-e^{-m x}}{x^{2}}\right) e^{i p \cdot x}=4 \pi\left[m-\frac{\pi p}{2}+p \arctan \frac{p}{m}\right],
$$

a non-vanishing surface term $4 \pi m$ appears. However, if we perform the Fourier transform according to the original idea of differential regularization [[f], this surface term is usually discarded. So we see that the surface terms are very important in Chern-Simons type theories since they are usually finite [17 19] at least at the one-loop level [20], and thus the nonvanishing surface term would result in an ambiguity in defining the finite renormalization. As we know, in a finite theory, the $\beta$-function and anomalous dimensions of each field vanish, the renormalization group equation is trivial and the only criterion for the equivalence among different renormalization conditions is that all the regularization schemes preserving the fundamental symmetry such as gauge invariance should give the same gauge invariant radiative corrections, so that the finite renormalization ambiguity is a serious problem. Therefore, we believe that in this respect differential regularization, thanks to its nature, provides with a better understanding of the renormalization ambiguity in Chern-Simons type theories. 


\section{ACKNOWLEDGMENTS}

The support of the Academy of Finland is greatly acknowledged. W.F.C. thanks the World Laboratory, Switzerland, for financial support. We thank M. Asorey and F. Ruiz Ruiz for their useful discussions on Chern-Simons theory and especially Z.Y. Zhu for his help in understanding the differential regularization. H.C.L. is partially supported by grant 85-2112-M-008-011 from the National Science Council, ROC. 


\section{REFERENCES}

[1] D.Z. Freedman, K. Johnson and J.I. Latorre, Nucl. Phys. B371 (1992) 353.

[2] P.E. Haagensen and J.I. Latorre, Phys. Lett. B283 (1992) 293.

[3] P.E. Haagensen, Mod. Phys. Lett. A7 (1992) 893.

[4] P.E. Haagensen and J.I. Latorre, Ann. Phys. 221 (1993) 77.

[5] R. Munoz-Tapia, Phys. Lett. B295 (1992) 95.

[6] G. Dunne and N. Rius, Phys. lett. B293 (1992) 367.

[7] D.Z. Freedman, K. Johnson, R. Munoz-Tapia and X. Vilasis-Cardona, Nucl. Phys. B395 (1993) 454 .

[8] V.A. Smirnov, Nucl. Phys. B427 (1994) 325; Z. Phys. C67 (1995) 531.

[9] J. Erdmenger and H. Osborn, Nucl. Phys. B483 (1997) 431.

[10] S. Deser, R. Jackiw and S. Templeton, Phys. Rev. Lett. 48 (1982) 975; Phys. Rev. D23 (1981) 2291; Ann. Phys. 140 (1982) 372.

[11] E. Guadagnini, M. Matellini and M. Mintchev, Phys. Lett. B227 (1989) 111; W. Chen, G. W. Semenoff and Y.S. Wu, Mod. Phys. Lett. A5 (1990) 1833; Phys. Rev. D46 (1992) 5521; L. Alvarez-Gaumé, J.M.F. Labastida and A.F. Ramallo, Nucl. Phys. B334 (1990) 103; M. Asorey and F. Falceto, Phys. Lett. B241 (1990) 31; C. P. Martin, Phys. Lett. B241 (1990) 513; G. Giavarini, C.P. Martin and F. Ruiz Ruiz, Nucl. Phys. B381 (1992) 222, Phys. Rev. D47 (1993) 5536; W. F. Chen and Z.Y. Zhu, J. Phys. A27 (1994) 1781.

[12] M. Asorey, F. Falceto, J. L. López and G. Luzón, Phys. Rev. D49 (1994) 5377; Nucl. Phys. B429 (1994) 344.

[13] M. Leblanc and M.T. Thomaz, Phys. Lett. B281 (1992) 259.

[14] C. P. Martin and F. Ruiz Ruiz, Nucl. Phys. B436 (1995) 545. 
[15] M. Asorey and F. Falceto, Phys. Rev. D54 (1996) 5290.

[16] W.F. Chen, H.C. Lee and Z.Y. Zhu, Phys. Rev. D55 (1997) 3664.

[17] A. Blasi and R. Collina, Nucl. Phys. B345 (1990) 472.

[18] F. Delduc, O. Piguet, C. Lucchesi and S.P. Sorella, Nucl. Phys. B346 (1990) 313.

[19] A. Blasi, N. Maggiore and S.P. Sorella, Phys. Lett. B285 (1992) 54.

[20] E.R. Speer, J. Math. Phys. 15 (1974) 1. 